\title{
Analysis of Sera from Cattle Suspected for Brucellosis by Agglutination Tests and ELISA
}

\author{
Anju Mohan and Hari Mohan Saxena* \\ Department of Veterinary Microbiology, College of Veterinary Science, GADVASU, \\ Ludhiana-141004, Punjab, India \\ *Corresponding author
}

\section{A B S T R A C T}

Keywords Brucellosis, RBPT, STAT, MAT and ELISA

Article Info

Accepted: 04 October 2018 Available Online: 10 November 2018
Brucellosis is a major bacterial zoonosis of global importance. Bovine brucellosis is endemic in all states of India. However, the exact extent of the disease occurrence in the unorganized herds is not known. The present study was undertaken for serological diagnosis of brucellosis in cattle from a local gaushala (cow shelter) employing common agglutination tests RBPT, STAT and MAT and ELISA. Out of 56 samples from cattle from the Gaushala, $37.5 \%$ samples by RBPT, $64.28 \%$ each by STAT and MAT and $49.23 \%$ samples by ELISA, respectively were found to be positive for anti-Brucella antibodies. Among the 29 suspected serum samples from the field cattle, samples found to be positive for anti-Brucella antibodies were $100 \%$ by RBPT, $79.31 \%$ by MAT and $89.65 \%$ by ELISA, respectively. Titers of anti-Brucella antibodies in the sera of cattle from Gaushala were found to be $1.747 \pm 0.45,1.935 \pm 0.62$ and $0.943 \pm 0.46$ by STAT, MAT and ELISA, respectively, while those in cattle from the field were found to be $1.577 \pm 0.60$ and $0.783 \pm 0.59$ by MAT and ELISA, respectively.

\section{Introduction}

Brucellosis is a major bacterial zoonosis of global importance. It occurs worldwide but is much controlled in developed countries by routine screening of domestic animals and vaccination program. Clinical disease is still common in Middle East, Asia, Africa, South and Central America, the Mediterranean Basin, and the Caribbean. About 500, 000 cases of human brucellosis are estimated to occur worldwide every year. It causes heavy economic loss to the animal industry through abortion, delayed conception, and temporary or permanent infertility in the affected animals
(Kollannur et al., 2007). In Punjab, overall $17.7 \%$ prevalence of brucellosis was reported in cattle and buffaloes (Jain et al., 2013; Pandeya et al., 2013). Brucellosis in animals is clinically characterized by late-term abortions and retention of placenta in females and orchitis and epididymitis in males, with excretion of organisms in semen, uterine discharges, and in milk (Jain et al., 2013; Godfroid et al., 2013). Once infected, the animal may continue to shed bacteria and remains a source of infection to others for long period (Pandeya et al., 2013). Antibodies induced by vaccination interfere in serological diagnosis of brucellosis. Bovine brucellosis is 
endemic in all states of India. In India, the occurrence of brucellosis is to the extent of $10 \%$ in the marginal herds and $50 \%$ in organized farms, and the socio-economic impact of the disease was estimated to run over Rs. 500 crores annually. However, little data are available in the published literature on antibody titers due to natural infection in cattle in unorganized herds, gaushalas etc. from different regions of India. The present study was, therefore, undertaken for serological analysis of brucellosis in cattle at a gaushala.

\section{Materials and Methods}

\section{Collection of samples}

Blood samples from cattle were collected from the gaushala, Ludhiana, Punjab. About $10 \mathrm{ml}$ of blood was collected aseptically from the jugular vein of the animal. Serum was collected by centrifuging the clotted blood at $3000 \mathrm{rpm}$ for 15 minutes.

\section{Rose Bengal Plate Agglutination Test (RBPT)}

Equal volumes (10 $\mu$ l each) of RBPT coloured antigen (Punjab Veterinary Vaccine Institute, Ludhiana) and test serum were mixed on a clean glass slide (Morgan et al., 1978) with the help of a sterilized toothpick. The slide was observed for $4 \mathrm{~min}$ for the formation of clumps. The formation of clumps was considered a positive test while the absence of clear clumps was considered a negative reaction.

\section{Standard Tube Agglutination Test (STAT)}

The scheme of dilution of sera followed for STAT is depicted in Table 1. Eight agglutination tubes were placed in a rack. 0.8 $\mathrm{ml}$ of $0.5 \%$ carbol saline was added to the first tube of the series. $0.5 \mathrm{ml}$ of carbol saline was added to all the other tubes except in tube no.
06, 07 and 08 which contained 1.25, 1.50 and $1.75 \mathrm{ml}$ of carbol saline respectively.

$0.2 \mathrm{ml}$ serum was added to the first tube and the contents were mixed. $0.5 \mathrm{ml}$ of Brucella abortus plain antigen (Punjab Veterinary Vaccine Institute, Ludhiana) was then added to tube numbers 1 to 9 , giving a final dilution of 1: 10, 1:20,1: 40 and so on. To the tubes 10, 11 and 12, which were kept as controls, $0.75,0.50$ and $0.25 \mathrm{ml}$ respectively of Brucella abortus plain antigen was added. These tubes were incubated at $37^{\circ} \mathrm{C}$ for 24 hours and the results were read.

The results were compared with the antigen control tube showing 50\% agglutination. The highest serum dilution showing $50 \%$ agglutination was taken as the end point for the titre serum. A titre of 1:40 or above was considered positive.

\section{Microtitre Plate Agglutination Test (MAT)}

MAT was performed as per the standard method (Williams and Whittemore, 1971).

\section{Test protocol}

Serum samples were serially diluted two fold in a final volume of $100 \mu \mathrm{l}$ in 96 well Ubottom microtitre plate (Tarsons).

Equal volume of $100 \mu 1$ Brucella abortus plain antigen (Punjab Veterinary Vaccine Institute, Ludhiana) was added to each well. Negative control well containing $100 \mu \mathrm{l}$ of sterilized Normal Saline Solution and $100 \mu \mathrm{l}$ of the antigen was also kept.

The plate was covered with a lid and incubated at $37^{\circ} \mathrm{C}$ for $24 \mathrm{hr}$ followed by incubation at $4^{\circ} \mathrm{C}$ for 1 hour.

The formation of matt signified agglutination while button formation was indicative of a 
negative reaction. Titres ( $\log _{10}$ values) were recorded as the reciprocal of the highest dilution of the serum giving at least 50percent agglutination.

\section{Enzyme Linked Immunosorbent Assay (ELISA)}

Serum samples of cattle were tested using Idexx Brucellosis Serum ELISA test kit.

\section{Principle}

Microtiter plates are supplied precoated with inactivated antigen. Dilutions of the samples to be tested are incubated in the wells of these plates. Any antibody specific for B. abortus binds to the antigen in the wells and forms an antigen/ antibody complex on the plate well surface. Unbound material is removed from the wells by washing. A peroxidase- labeled anti ruminant IgG Conjugate is added, which binds to the ruminant antibodies complexed with the $B$. abortus antigen. Unbound Conjugate is removed by washing and the TMB substrate is added to the wells. The degree of the colour that develops (optical density measured at $450 \mathrm{~nm}$ ) is directly proportional to the amount of antibody specific for $B$. abortus present in the sample. The diagnostic relevance of the result is obtained by comparing the optical density (OD) that develops in wells containing the samples with the OD from wells containing the Positive Control.

\section{Test procedure}

All reagents must be allowed to come to $18-26$ ${ }^{\circ} \mathrm{C}$ before use. Reagents should be mixed by gentle swirling or vortexing.

Dispense $90 \mu \mathrm{l}$ Wash Solution into each well of the microtiter plate.

Add $10 \mu \mathrm{l}$ of the undiluted serum samples and Controls into the appropriate wells of the microtiter plate. Final dilution $=1: 10$. Mix the contents within each well by gently shaking the microtiter plate briefly (a microtiter plate shaker can be used).

Cover the microtiter plate with a lid and incubate for 60 minutes at $37{ }^{\circ} \mathrm{C}$ in a humid chamber.

Wash each well with approximately $300 \mu \mathrm{l}$ Wash Solution three times. Aspirate liquid contents of all wells after each wash. Following the final aspiration, firmly tap residual wash fluid from each plate onto absorbent material. Avoid plate drying between washes and prior to the addition of the next reagent.

Dispense $100 \mu$ l Conjugate into each well.

Cover and incubate the microtiter plate for 60 minutes at $37{ }^{\circ} \mathrm{C}$ in a humid chamber.

Wash each well and aspirate the liquid contents of all wells after each well.

Dispense $100 \mu \mathrm{l}$ of TMB substrate into each well.

Incubate the Substrate at $18-26^{\circ} \mathrm{C}$ for 15 minutes.

Stop the colour reaction by adding $100 \mu$ l Stop solution per well. The Stop Solution should be dispensed in the same order and at the same speed as the substrate.

Read the results using photometer at a wavelength of $450 \mathrm{~nm}$.

To validate the assay, the optical density (OD) of the Positive Control should not exceed 2.000 and the OD of the Negative Control should not exceed 0.5000. The difference between the Positive and the Negative Control must be greater than or equal to 0.3000 . 
The plates should be read within two hours after the addition of stop solution.

\section{Calculations}

The OD of duplicates was averaged.

The OD of the Positive Control (PCx) and the OD of the samples were corrected by subtracting the OD of the Negative Control (NCx).

Positive Control corrected OD: PCX- NCX

Sample corrected OD: Sample A450- NCx

$\mathrm{S} / \mathrm{P}$ ratio for each sample: $\mathrm{S} / \mathrm{P} \%=100 \mathrm{x}$ (Sample A 450- NCx / PCx- NCx)

\section{Interpretation of results}

$\mathrm{S} / \mathrm{P}$ ratio less than $80 \%$ - Negative

$\mathrm{S} / \mathrm{P}$ greater than or equal to $80 \%$ - Positive

\section{Results and Discussion}

Out of 56 suspected samples from cattle from the Gaushala, samples found to be positive for anti-Brucella antibodies were $21(37.5 \%)$ by RBPT, 36 (64.28\%) each by STAT and MAT and $32(49.23 \%)$ by ELISA, respectively (Tables 2 and 3). Among the 29 suspected serum samples from the field cattle, samples found to be positive for anti-Brucella antibodies were $29(100 \%)$ by RBPT, 23 $(79.31 \%)$ by MAT and $26(89.65 \%)$ by ELISA, respectively (Tables 4 and 5). Titers of anti-Brucella antibodies in the sera of cattle from Gaushala were found to be $1.747 \pm 0.45$, $1.935 \pm 0.62$ and $0.943 \pm 0.46$ by STAT, MAT and ELISA, respectively (Table 6). Titers in the sera of cattle from the field were found to be $1.577 \pm 0.60$ and $0.783 \pm 0.59$ by MAT and ELISA, respectively (Table 7).
There was no significant difference between mean titers by STAT and MAT of Gaushala cattle and between mean MAT titers as well as between the ELISA titers of the two groups of animals, respectively.

Even though a number of antigenic components of Brucella have been characterized, the antigen that dominates the antibody response is the lipopolysaccharide (LPS). Numerous outer and inner membrane, cytoplasmic, and periplasmic protein antigens have also been characterized.

Some are recognized by the immune system during infection and are potentially useful in diagnostic tests. The L7/L12 ribosomal proteins are important in stimulating cellmediated responses (Oliveira and Splitter, 1994).

Immune response of host to Brucella infection is mediated through both humoral and cellmediated immunity (Skendros and Boura, 2013). The role of humoral immunity against intracellular bacterial infections is limited and not protective. Antibody-mediated opsonization by Igs (IgM, IgG1, IgG2a, and IgG3) enhances phagocytic uptake of bacteria, limiting the level of initial infection with Brucella but has little effect on the intracellular course of Brucella infection (Bellaire et al., 2005; Baldwin and Goenka, 2006).

As evident from our present data, infected animals had high titers of antibodies. However, the high titers do not indicate whether these are protective antibodies due to vaccine or acute response to infection in the absence of a differentiation of infected from vaccinated animals (DIVA) assay or whether they are of any relevance to prognosis. 
Table.1 Scheme of dilution of sera for STAT

\begin{tabular}{|c|c|c|c|c|}
\hline $\begin{array}{l}\text { Tube } \\
\text { No. }\end{array}$ & $\begin{array}{l}\text { Carbol saline } \\
\text { (ml) }\end{array}$ & Test serum(ml) & $\begin{array}{c}\text { B. abortus plain } \\
\text { antigen (ml) }\end{array}$ & $\begin{array}{l}\text { Final } \\
\text { dilution }\end{array}$ \\
\hline 1 & 0.8 & 0.2 & 0.5 & $1: 10$ \\
\hline 2 & 0.5 & \multirow{7}{*}{$\begin{array}{l}\text { Serial dilution was performed after } \\
\text { thorough mixing. } 0.5 \mathrm{ml} \text { of the contents } \\
\text { was transferred from tube no. } 1 \text { to the next } \\
\text { tube up to tube no. } 5 \text {. Finally } 0.5 \mathrm{ml} \text { of the } \\
\text { contents was discarded from tube no.5. }\end{array}$} & 0.5 & $1: 20$ \\
\hline 3 & 0.5 & & 0.5 & $1: 40$ \\
\hline 4 & 0.5 & & 0.5 & $1: 80$ \\
\hline 5 & 0.5 & & 0.5 & $1: 160$ \\
\hline 6 & 1.25 & & 0.75 & \\
\hline 7 & 1.50 & & 0.50 & \\
\hline 8 & 1.75 & & 0.25 & \\
\hline
\end{tabular}

Controls:

Tube No. 6- $25 \%$ agglutination

Tube No. 7- $50 \%$ agglutination

Tube No. 8- $75 \%$ agglutination

Table.2 Sera from cattle analysed for anti-Brucella antibodies by four serological tests

\begin{tabular}{|c|c|c|c|c|c|}
\hline S. No. & Sample number & RBPT & STAT & MAT & ELISA \\
\hline 1 & BS 1 & $\mathrm{~N}$ & $\mathrm{~N}$ & $\mathrm{~N}$ & $\mathrm{~N}$ \\
\hline 2 & BS 2 & $\mathrm{~N}$ & $\mathrm{~N}$ & $\mathrm{~N}$ & $\mathrm{~N}$ \\
\hline 3 & BS 3 & $\mathrm{~N}$ & $\mathrm{~N}$ & $\mathrm{~N}$ & $\mathrm{~N}$ \\
\hline 4 & BS 4 & $\mathrm{P}$ & $\mathrm{P}$ & $\mathrm{P}$ & $\mathrm{P}$ \\
\hline 5 & BS 5 & $\mathrm{~N}$ & $\mathrm{~N}$ & $\mathrm{~N}$ & $\mathrm{~N}$ \\
\hline 6 & BS 6 & $\mathrm{~N}$ & $\mathrm{~N}$ & $\mathrm{~N}$ & $\mathrm{~N}$ \\
\hline 7 & BS 7 & $\mathrm{~N}$ & $\mathrm{~N}$ & $\mathrm{~N}$ & $\mathrm{~N}$ \\
\hline 8 & BS 8 & $\mathrm{~N}$ & $\mathrm{~N}$ & $\mathrm{P}$ & $\mathrm{N}$ \\
\hline 9 & BS 9 & $\mathrm{~N}$ & $\mathrm{~N}$ & $\mathrm{P}$ & $\mathrm{N}$ \\
\hline 10 & BS 10 & $\mathrm{~N}$ & $\mathrm{~N}$ & $\mathrm{P}$ & $\mathrm{N}$ \\
\hline 11 & BS 11 & $\mathrm{P}$ & $\mathrm{P}$ & $\mathrm{P}$ & $\mathrm{P}$ \\
\hline 12 & BS 12 & $\mathrm{P}$ & $\mathrm{P}$ & $\mathrm{P}$ & $\mathrm{N}$ \\
\hline 13 & BS 13 & $\mathrm{P}$ & $\mathrm{P}$ & $\mathrm{P}$ & $\mathrm{P}$ \\
\hline 14 & BS 14 & $\mathrm{P}$ & $\mathrm{P}$ & $\mathrm{P}$ & $\mathrm{P}$ \\
\hline
\end{tabular}




\begin{tabular}{|c|c|c|c|c|c|}
\hline 15 & BS 15 & $\mathrm{~N}$ & P & $\mathrm{N}$ & $\mathrm{N}$ \\
\hline 16 & BS 16 & $\mathrm{P}$ & $\mathrm{P}$ & $\mathrm{P}$ & $\mathrm{P}$ \\
\hline 17 & BS 17 & $\mathrm{~N}$ & $\mathrm{P}$ & $\mathrm{N}$ & $\mathrm{P}$ \\
\hline 18 & BS 18 & $\mathrm{~N}$ & P & $\mathrm{P}$ & $\mathrm{N}$ \\
\hline 19 & BS 19 & $\mathrm{~N}$ & $\mathrm{~N}$ & $\mathrm{~N}$ & $\mathrm{~N}$ \\
\hline 20 & BS 20 & $\mathrm{~N}$ & $\mathrm{~N}$ & $\mathrm{~N}$ & $\mathrm{~N}$ \\
\hline 21 & BS 21 & $\mathrm{~N}$ & $\mathrm{P}$ & $\mathrm{P}$ & $\mathrm{N}$ \\
\hline 22 & BS 22 & $\mathrm{~N}$ & $\mathrm{~N}$ & $\mathrm{P}$ & $\mathrm{N}$ \\
\hline 23 & BS 23 & $\mathrm{~N}$ & $\mathrm{P}$ & $\mathrm{P}$ & $\mathrm{P}$ \\
\hline 24 & BS 24 & $\mathrm{~N}$ & $\mathrm{~N}$ & $\mathrm{~N}$ & $\mathrm{~N}$ \\
\hline 25 & BS 25 & $\mathrm{P}$ & $\mathrm{P}$ & $\mathrm{P}$ & $\mathrm{N}$ \\
\hline 26 & BS 26 & $\mathrm{P}$ & $\mathrm{P}$ & $\mathrm{P}$ & $\mathrm{P}$ \\
\hline 27 & BS 27 & $\mathrm{~N}$ & $\mathrm{P}$ & $\mathrm{P}$ & P \\
\hline 28 & BS 28 & $\mathrm{P}$ & $\mathrm{P}$ & $\mathrm{P}$ & $\mathrm{P}$ \\
\hline 29 & BS 29 & $\mathrm{P}$ & $\mathrm{P}$ & P & P \\
\hline 30 & BS 30 & $\mathrm{~N}$ & $\mathrm{P}$ & $\mathrm{N}$ & $\mathrm{P}$ \\
\hline 31 & BS 31 & $\mathrm{~N}$ & $\mathrm{~N}$ & $\mathrm{~N}$ & $\mathrm{~N}$ \\
\hline 32 & BS 32 & $\mathrm{~N}$ & P & $\mathrm{P}$ & P \\
\hline 33 & BS 33 & P & P & $\mathrm{P}$ & P \\
\hline 34 & BS 34 & $\mathrm{P}$ & $\mathrm{P}$ & $\mathrm{P}$ & $\mathrm{P}$ \\
\hline 35 & BS 35 & $\mathrm{~N}$ & P & P & P \\
\hline 36 & BS 36 & $\mathrm{~N}$ & $\mathrm{~N}$ & $\mathrm{~N}$ & $\mathrm{~N}$ \\
\hline 37 & BS 37 & $\mathrm{~N}$ & $\mathrm{P}$ & $\mathrm{N}$ & $\mathrm{P}$ \\
\hline 38 & BS 38 & $\mathrm{P}$ & $\mathrm{P}$ & $\mathrm{P}$ & $\mathrm{P}$ \\
\hline 39 & BS 39 & P & $\mathrm{P}$ & P & P \\
\hline 40 & BS 40 & $\mathrm{P}$ & $\mathrm{P}$ & $\mathrm{P}$ & $\mathrm{P}$ \\
\hline 41 & BS 41 & $\mathrm{~N}$ & $\mathrm{~N}$ & $\mathrm{P}$ & $\mathrm{P}$ \\
\hline 42 & BS 42 & $\mathrm{~N}$ & $\mathrm{P}$ & $\mathrm{P}$ & $\mathrm{P}$ \\
\hline 43 & BS 43 & $\mathrm{P}$ & $\mathrm{P}$ & $\mathrm{P}$ & $\mathrm{P}$ \\
\hline 44 & BS 44 & $\mathrm{~N}$ & $\mathrm{~N}$ & $\mathrm{~N}$ & $\mathrm{~N}$ \\
\hline$\overline{45}$ & BS 45 & $\mathrm{P}$ & P & P & P \\
\hline 46 & BS 46 & P & $\mathrm{P}$ & P & $\mathrm{P}$ \\
\hline$\overline{47}$ & BS 47 & $\mathrm{~N}$ & $\mathrm{~N}$ & $\mathrm{~N}$ & $\mathrm{~N}$ \\
\hline 48 & BS 48 & $\mathrm{~N}$ & $\mathrm{~N}$ & $\mathrm{~N}$ & $\mathrm{~N}$ \\
\hline$\overline{49}$ & BS 49 & $\mathrm{~N}$ & $\mathrm{P}$ & P & $\mathrm{P}$ \\
\hline 50 & BS 50 & $\mathrm{~N}$ & $\mathrm{P}$ & $\mathrm{P}$ & $\mathrm{P}$ \\
\hline 51 & BS 51 & P & P & P & P \\
\hline 52 & BS 52 & $\mathrm{~N}$ & $\mathrm{P}$ & $\mathrm{P}$ & $\mathrm{P}$ \\
\hline 53 & BS 53 & $\mathrm{~N}$ & P & $\mathrm{N}$ & $\mathrm{P}$ \\
\hline 54 & BS 54 & $\mathrm{~N}$ & $\mathrm{~N}$ & $\mathrm{~N}$ & $\mathrm{~N}$ \\
\hline 55 & BS 55 & $\mathrm{P}$ & $\mathrm{P}$ & $\mathrm{P}$ & $\mathrm{P}$ \\
\hline 56 & BS 56 & $\mathrm{P}$ & $\mathrm{P}$ & $\mathrm{P}$ & $\mathrm{P}$ \\
\hline
\end{tabular}


Table.3 Percentage of positive samples from Gaushala by RBPT, STAT, MAT and ELISA

\begin{tabular}{l|l|} 
Serological test \\
\hline RBPT \\
\hline STAT \\
\hline MAT \\
\hline ELISA
\end{tabular}

\begin{tabular}{|l|l|l|}
\hline \multicolumn{2}{|c|}{ Number of samples } & Percent positive \\
\hline 21 & Positive & Negative \\
\hline 36 & 35 & 37.5 \\
\hline 36 & 20 & 64.28 \\
\hline 32 & 20 & 64.28 \\
\hline
\end{tabular}

Table.4 Sera from field cattle positive for Brucellosis by RBPT, MAT and ELISA

\begin{tabular}{|c|}
\hline S.no. \\
\hline 1 \\
\hline 2 \\
\hline 3 \\
\hline 4 \\
\hline 5 \\
\hline 6 \\
\hline 7 \\
\hline 8 \\
\hline 9 \\
\hline 10 \\
\hline 11 \\
\hline 12 \\
\hline 13 \\
\hline 14 \\
\hline 15 \\
\hline 16 \\
\hline 17 \\
\hline 18 \\
\hline 19 \\
\hline 20 \\
\hline 21 \\
\hline 22 \\
\hline 23 \\
\hline 24 \\
\hline 26 \\
\hline 27 \\
\hline 28 \\
\hline
\end{tabular}

\begin{tabular}{|c|r|}
\hline Animal no. & RB \\
\hline FS 3 & P \\
\hline FS 4 & P \\
\hline FS 5 & P \\
\hline FS 10 & P \\
\hline FS 15 19 & P \\
\hline S 1 & P \\
\hline S 2 & P \\
\hline S 3 & P \\
\hline S 4 & P \\
\hline S 6 & P \\
\hline S 7 & P \\
\hline S 12 & P \\
\hline S 17 & P \\
\hline S 18 & P \\
\hline S 19 & P \\
\hline S 20 & P \\
\hline S 24 & P \\
\hline S 33 & P \\
\hline S 34 & P \\
\hline S 38 & P \\
\hline S 39 & P \\
\hline S 40 & P \\
\hline S 43 & P \\
\hline S 44 & P \\
\hline S 47 & P \\
\hline S 49 & P \\
\hline S 51 & P \\
\hline S 53 & P \\
\hline
\end{tabular}

\begin{tabular}{|c|c|}
\hline MAT & ELISA \\
\hline P & P \\
\hline P & P \\
\hline P & P \\
\hline P & P \\
\hline P & P \\
\hline P & N \\
\hline N & P \\
\hline P & P \\
\hline P & P \\
\hline N & N \\
\hline P & P \\
\hline P & P \\
\hline P & P \\
\hline P & P \\
\hline P & P \\
\hline P & P \\
\hline N & P \\
\hline P & P \\
\hline P & P \\
\hline P & P \\
\hline N & P \\
\hline P & P \\
\hline N & P \\
\hline P & P \\
\hline
\end{tabular}

Table.5 Percentage of positive samples from field cattle by RBPT, MAT and ELISA

\begin{tabular}{l} 
Serological test \\
\hline RBPT \\
\hline MAT \\
\hline ELISA
\end{tabular}

\begin{tabular}{|l|l|}
\multicolumn{2}{|c|}{ Number of } \\
\hline & Positive \\
\hline 29 & \\
\hline 23 & \\
\hline 26 & \\
\hline
\end{tabular}

Percent positive

\begin{tabular}{|l|l|}
\hline & Negative \\
\hline 0 & \\
\hline 6 & \\
\hline 3 & \\
\hline
\end{tabular}

100

79.31

89.65 
Table.6 Titers of anti-Brucella antibodies in sera of cattle from Gaushala

\begin{tabular}{|c|c|c|c|c|}
\hline S. No. & Sample number & STAT & MAT & ELISA \\
\hline 1 & BS 1 & 1.3010 & 1.0000 & 0.0684 \\
\hline 2 & BS 2 & 1.3010 & 1.0000 & 0.4764 \\
\hline 3 & BS 3 & 1.3010 & 1.3010 & 0.0758 \\
\hline 4 & BS 4 & 2.2041 & 2.5051 & 1.3222 \\
\hline 5 & BS 5 & 1.0000 & 1.0000 & 0.8646 \\
\hline 6 & BS 6 & 1.3010 & 1.3010 & 0.0736 \\
\hline 7 & BS 7 & 1.0000 & 1.0000 & 0.7809 \\
\hline 8 & BS 8 & 1.3010 & 2.5051 & 0.6319 \\
\hline 9 & BS 9 & 1.3010 & 2.2041 & 0.1001 \\
\hline 10 & BS 10 & 1.3010 & 2.5051 & 0.0776 \\
\hline 11 & BS 11 & 2.2041 & 2.5051 & 1.1153 \\
\hline 12 & BS 12 & 2.2041 & 2.5051 & 1.0032 \\
\hline 13 & BS 13 & 2.2041 & 2.5051 & 1.0361 \\
\hline 14 & BS 14 & 2.2041 & 2.5051 & 1.7271 \\
\hline 15 & BS 15 & 1.6020 & 1.3010 & 0.8589 \\
\hline 16 & BS 16 & 2.2041 & 2.5051 & 1.4086 \\
\hline 17 & BS 17 & 2.2041 & 1.3010 & 1.0518 \\
\hline 18 & BS 18 & 2.2041 & 2.5051 & 0.3805 \\
\hline 19 & BS 19 & 1.3010 & 1.0000 & 0.0979 \\
\hline 20 & BS 20 & 1.0000 & 1.0000 & 0.7397 \\
\hline 21 & BS 21 & 1.9030 & 2.5051 & 0.7407 \\
\hline 22 & BS 22 & 1.3010 & 2.5051 & 0.8679 \\
\hline 23 & BS 23 & 1.9030 & 2.5051 & 1.1306 \\
\hline 24 & BS 24 & 1.0000 & 1.0000 & 0.1505 \\
\hline 25 & BS 25 & 2.2041 & 2.5051 & 0.9017 \\
\hline 26 & BS 26 & 2.2041 & 2.5051 & 1.184 \\
\hline 27 & BS 27 & 1.9030 & 2.5051 & 1.154 \\
\hline 28 & BS 28 & 2.2041 & 2.5051 & 1.587 \\
\hline 29 & BS 29 & 2.2041 & 2.2041 & 0.954 \\
\hline 30 & BS 30 & 1.6020 & 1.3010 & 0.888 \\
\hline 31 & BS 31 & 1.3010 & 1.0000 & 0.344 \\
\hline 32 & BS 32 & 1.6020 & 2.2041 & 0.912 \\
\hline 33 & BS 33 & 2.2041 & 2.2041 & 1.394 \\
\hline 34 & BS 34 & 2.2041 & 2.5051 & 1.547 \\
\hline 35 & BS 35 & 1.9030 & 1.9030 & 1.315 \\
\hline 36 & BS 36 & 1.3010 & 1.3010 & 0.813 \\
\hline 37 & BS 37 & 1.9030 & 1.3010 & 1.129 \\
\hline 38 & BS 38 & 2.2041 & 2.5051 & 1.462 \\
\hline 39 & BS 39 & 2.2041 & 2.5051 & 1.447 \\
\hline 40 & BS 40 & 2.2041 & 2.5051 & 1.077 \\
\hline 41 & BS 41 & 1.3010 & 1.6020 & 1.295 \\
\hline 42 & BS 42 & 1.9030 & 1.9030 & 1.411 \\
\hline 43 & BS 43 & 2.2041 & 2.5051 & 1.557 \\
\hline 44 & BS 44 & 1.0000 & 1.0000 & 0.050 \\
\hline 45 & BS 45 & 2.2041 & 2.5051 & 1.383 \\
\hline 46 & BS 46 & 2.2041 & 2.5051 & 1.480 \\
\hline 47 & BS 47 & 1.0000 & 1.0000 & 0.619 \\
\hline 48 & BS 48 & 1.3010 & 1.3010 & 0.860 \\
\hline 49 & BS 49 & 1.6020 & 1.9030 & 1.106 \\
\hline 50 & BS 50 & 1.9030 & 2.2041 & 1.300 \\
\hline 51 & BS 51 & 2.2041 & 2.5051 & 1.196 \\
\hline 52 & BS 52 & 1.9030 & 1.9030 & 1.257 \\
\hline 53 & BS 53 & 1.6020 & 1.3010 & 1.273 \\
\hline 54 & BS 54 & 1.0000 & 1.3010 & 0.571 \\
\hline 55 & BS 55 & 2.2041 & 2.5051 & 1.158 \\
\hline 56 & BS 56 & 2.2041 & 2.5051 & 1.416 \\
\hline \multicolumn{2}{|c|}{ Mean + SD } & $1.747 \pm 0.45$ & $1.935 \pm 0.62$ & $0.943 \pm 0.46$ \\
\hline
\end{tabular}


Table.7 Anti-Brucella antibody titers in sera of field cattle by MAT and ELISA

\begin{tabular}{|c|c|c|c|}
\hline S. no. & Sample no. & MAT & ELISA \\
\hline 1 & FS 1 & 1.0000 & 0.063 \\
\hline 2 & FS 2 & 1.3010 & 0.419 \\
\hline 3 & FS 3 & 2.5051 & 1.104 \\
\hline 4 & FS 4 & 1.9030 & 1.213 \\
\hline 5 & FS 5 & 2.5051 & 1.238 \\
\hline 6 & FS 6 & 1.3010 & 0.246 \\
\hline 7 & FS 7 & 1.6020 & 0.061 \\
\hline 8 & FS 8 & 1.3010 & 1.103 \\
\hline 9 & FS 9 & 1.0000 & 0.053 \\
\hline 10 & FS 10 & 1.6020 & 1.344 \\
\hline 11 & FS 11 & 1.0000 & 0.054 \\
\hline 12 & FS 12 & 1.0000 & 0.058 \\
\hline 13 & FS 13 & 1.0000 & 0.055 \\
\hline 14 & FS 14 & 1.3010 & 0.059 \\
\hline 15 & FS 15 & 2.5051 & 1.420 \\
\hline 16 & FS 16 & 2.2041 & 0.115 \\
\hline 17 & FS 17 & 1.6020 & 0.061 \\
\hline 18 & FS 18 & 2.5051 & 0.076 \\
\hline 19 & FS19 & 2.5051 & 1.537 \\
\hline 20 & FS 20 & 2.5051 & 0.126 \\
\hline 21 & S 1 & 1.6020 & 0.105 \\
\hline 22 & S 2 & 1.3010 & 1.354 \\
\hline 23 & S 3 & 1.0000 & 0.218 \\
\hline 24 & S 4 & 2.2041 & 1.664 \\
\hline 25 & S 5 & 1.3010 & 0.3252 \\
\hline 26 & S 6 & 2.2041 & 1.360 \\
\hline 27 & S 7 & 1.3010 & 0.862 \\
\hline 28 & S 8 & 1.6020 & 0.099 \\
\hline 29 & S 9 & 1.0000 & 0.186 \\
\hline 30 & S 10 & 1.0000 & 0.4178 \\
\hline 31 & S 11 & 1.6020 & 0.160 \\
\hline 32 & S 12 & 2.5051 & 1.444 \\
\hline 33 & S 13 & 1.3010 & 1.324 \\
\hline 34 & S 14 & 1.0000 & 0.4317 \\
\hline 35 & S 15 & 1.0000 & 0.416 \\
\hline 36 & S 16 & 1.6020 & 0.5603 \\
\hline 37 & S 17 & 2.5051 & 1.688 \\
\hline 38 & S 18 & 2.2041 & 1.607 \\
\hline 39 & S 19 & 2.5051 & 1.536 \\
\hline 40 & S 20 & 2.5051 & 1.5642 \\
\hline
\end{tabular}




\begin{tabular}{|c|c|c|c|}
\hline 41 & S 21 & 1.0000 & 0.065 \\
\hline 42 & S 22 & 1.0000 & 1.076 \\
\hline 43 & S 23 & 1.0000 & 0.0715 \\
\hline 44 & S 24 & 2.5051 & 1.420 \\
\hline 45 & S 25 & 1.0000 & 0.4512 \\
\hline 46 & S 26 & 1.0000 & 1.2029 \\
\hline 47 & S 27 & 1.0000 & 1.1594 \\
\hline 48 & S 28 & 1.3010 & 1.297 \\
\hline 49 & S 29 & 1.3010 & 0.6211 \\
\hline 50 & S 30 & 1.0000 & 0.319 \\
\hline 51 & S 31 & 1.3010 & 1.088 \\
\hline 52 & S 32 & 1.0000 & 0.128 \\
\hline 53 & S 33 & 2.5051 & 1.558 \\
\hline 54 & S 34 & 1.3010 & 1.612 \\
\hline 55 & S 35 & 1.0000 & 1.158 \\
\hline 56 & S 36 & 1.0000 & 0.065 \\
\hline 57 & S 37 & 1.0000 & 0.525 \\
\hline 58 & S 38 & 1.6020 & 1.498 \\
\hline 59 & S 39 & 1.9030 & 0.978 \\
\hline 60 & S 40 & 1.6020 & 1.304 \\
\hline 61 & S 41 & 1.0000 & 1.006 \\
\hline 62 & S 42 & 1.0000 & 0.056 \\
\hline 63 & S 43 & 2.2041 & 1.252 \\
\hline 64 & S 44 & 1.3010 & 0.981 \\
\hline 65 & S 45 & 1.0000 & 0.106 \\
\hline 66 & S 46 & 1.0000 & 0.697 \\
\hline 67 & S 47 & 2.5051 & 1.292 \\
\hline 68 & S 48 & 1.3010 & 0.062 \\
\hline 69 & S 49 & 1.0000 & 1.371 \\
\hline 70 & S 50 & 2.2041 & 1.518 \\
\hline 71 & S 51 & 2.5051 & 1.458 \\
\hline 72 & S 52 & 1.0000 & 0.055 \\
\hline 73 & S 53 & 2.5051 & 1.489 \\
\hline 74 & S 54 & 2.5051 & 1.324 \\
\hline \multicolumn{2}{|c|}{ Mean \pm SD } & $1.577 \pm 0.60$ & $0.783 \pm 0.59$ \\
\hline
\end{tabular}

The different titers observed in the same animals by agglutination assays (STAT, MAT, and IHA) and iELISA can be reconciled with the fact that these assays target antigens of different nature, i.e., agglutination assays are directed toward particulate antigens, whereas ELISA detects immune response to soluble antigens. ELISA is generally used to detect IgG antibodies (Long et al., 1986). In brucellosis, specific IgM antibodies dominate during the acute phase of the disease (Smits et al., 2003). Specific IgG antibodies are present in the serum of patients at later stages of the illness and in the serum of relapsing patients (Ariza et al., 1992). ELISA is used to discriminate between the presence of specific IgM and IgG antibodies 
and to roughly assess the stage of illness (Smits and Kadri, 2005).

In many countries, STAT is the routine diagnostic test for human and animal brucellosis. It has been reported (Hassanain and Ahmed, 2012) that STAT has a greater accuracy than that of the RBPT $(93.3 \%$ and $76.6 \%$, respectively). Ina study (Sareyyupoglu et al., 2010), Brucella antibodies were investigated in bovine sera by RBPT, serum agglutination test, MAT, and 2-mercaptoethanol MAT, and MAT was determined as a fast, reliable, and economic test. On evaluation of canine brucellosis by MAT, it was shown (Kimura et al., 2008) that MAT was more sensitive, simpler to perform, and easier than tube agglutination test. A study (Versilova et al., 1974) has shown that the use of sheep erythrocytes sensitized with a specific LPS antigen in the IHA test provided a specific method, which is more sensitive than the agglutination test.

In a study by (Ghodasara et al., 2010), STAT and iELISA were compared for detection of Brucella antibodies in cows and buffaloes. The seropositivity was found highest by iELISA (25\%) followed by STAT $(14.45 \%)$. iELISA, RBPT, MAT, and PCR were evaluated (Malik et al., 2013) for diagnosis of brucellosis in buffaloes, and it was concluded that iELISA detected more samples as positive among these tests.

Among the suspected serum samples from cattle from the Gaushala, 37.5\% samples by RBPT, $64.28 \%$ each by STAT and MAT and $49.23 \%$ samples by ELISA, respectively were found to be positive for anti-Brucella antibodies. Among the serum samples from the field cattle, samples found to be positive for anti-Brucella antibodies were $100 \%$ by RBPT, $79.31 \%$ by MAT and $89.65 \%$ by ELISA, respectively. Titers in the sera of cattle from Gaushala were $1.747 \pm 0.45$, $1.935 \pm 0.62$ and $0.943 \pm 0.46$ by STAT, MAT and ELISA, respectively. While those in cattle from the field were $1.577 \pm 0.60$ and $0.783 \pm 0.59$ by MAT and ELISA, respectively.

\section{Conflict of interest}

The authors declare that they have no competing interests.

\section{Acknowledgements}

We are thankful to the Veterinary Officer in charge and the staff of the gaushala for cooperation and help.

\section{References}

Ariza, J., Pellicer, T., Pallares, R., Foz, A. and Gudiol, F. (1992) Specific antibody profile in human brucellosis. Clin. Infect. Dis., 14: 131-140.

Baldwin, C.L. and Goenka, R. (2006) Host immune responses to the intracellular bacterium Brucella: Does the bacterium instruct the host to facilitate chronic infection? Crit. Rev. Immunol., 26: 407442.

Bellaire, B.H., Roop, R.M. 2nd. and Cardelli, J.A. (2005) Opsonized virulent Brucella abortus replicates within nonacidic, endoplasmic reticulum-negative, LAMP1-positive phagosomes in human monocytes. Infect. Immun., 73: 37023713.

Ghodasara, S., Roy, A., Rank, D.N. and Bhander, B.B. (2010) Identification of Brucella species from animals with reproductive disorders by polymerase chain reaction assay. Buffalo Bull., 29(2): 98-108.

Godfroid, J., Garin-Bastuji, B., Saegerman, C. and Blasco, J.M. (2013) Brucellosis in terrestrial wildlife. Sci. Tech. Rev. Off. Int. Epiz., 32(1): 27-42.

Hassanain, N.A. and Ahmed, W.M. (2012) Efficacy of serological tests in comparison with PCR for diagnosis of brucellosis. World J. Med. Sci., 7(4): 243247.

Jain, U., Bisht, B., Sahzad, Pragati. and Dwivedi, K. (2013) Outbreak of brucellosis in buffaloes aborted in a village Mahuan, district Mainpuri, UP, 
India - A case report. Vet. World, 6(1): 51-52.

Kimura, M., Imaoka, K., Suzuki, M., Kamiyama, T. and Yamada, A. (2008) Evaluation of a microplate agglutination test (MAT) for serological diagnosis of canine brucellosis. J. Vet. Med. Sci., 70(7): 707-709.

Kollannur, J.D., Rathore, R. and Chauhan, R.S. (2007) Epidemiology and economics of brucellosis in animals and its zoonotic significance. Proceedings of XIII International Congress in Animal Hygiene. International Society for Animal Hygiene. P 466-468.

Long, G.Y., Liang, H.Z. and Liu, J.S. (1986) Immunization of rabbits against pasteurellosis. Chin. J. Vet. Sci. Technol., 2: 3-7.

Malik, R., Gupta, M.P., Sidhu, P.K., Filia, G., Saxena, H.M. and Shafi, T.A. (2013) Comparative evaluation of indirect enzyme linked immunosorbent assay, Rose Bengal plate test, microagglutination test and polymerase chain reaction for diagnosis of brucellosis in buffaloes. Turk. J. Vet. Anim. Sci., 37: 306-310.

Morgan, W.J., Mackinnon, D.T., Gill, K.P.W., Gower, S.G.M. and Norris, P.I.W. (1978) Brucellosis Diagnosis: Standard Laboratory Techniques Report Series No. 1. MAFF, Weybridge, England.

Oliveira, S.C. and Splitter, G.A. (1994) Subcloning and expression of Brucella abortus L7/L12 ribosomal gene and Tlymphocyte recognition of the recombinant protein. Infect. Immun., 62: 5201-5204.
Pandeya, Y.R., Joshi, D.D., Dhakal, S., Ghimire, L., Mahato, B.R., Chaulagain, S., Satyal, R.C. and Sah, S.K. (2013) Seroprevalence of brucellosis in different animal species of Kailali district Nepal. Int. J. Infect. Microbiol., 2(1): 22-25.

Sareyyupoglu, B., Cantekin, Z. and Mustak, H.K. (2010) Investigation of Brucella antibodies in bovine sera by rose Bengal plate test (RBPT), serum agglutination test (SAT), micro-agglutination test (MAT) and 2-mercaptoethanol- microagglutination (2-ME-MAT) test. Ankara Univ. Vet. Fak. Derg., 57: 157-160.

Skendros, P. and Boura, P. (2013) Immunity to brucellosis. Sci. Tech. Rev. Off. Int. Epiz., 32(1): 137-147.

Smits, H.L. and Kadri, S.M. (2005) Brucellosis in India: A deceptive infectious disease. Indian J. Med. Res., 122(5): 375-384.

Smits, H.L., Abdoel, T.H., Solera, J., Clavijo, E. and Diaz, R. (2003) Immunochromatographic Brucella-specific immunoglobulin $\mathrm{M}$ and $\mathrm{G}$ lateral flow assays for rapid serodiagnosis of human brucellosis. Clin. Diag. Lab. Immunol., 10: 1141-1146.

Versilova, P.A., Cernyseva, M.I., Aslanjan, R.G. and Knjazeva, E.N. (1974) Diagnosis of human and animal brucellosis by the indirect haemagglutination test. Bull. World Health Organ., 51: 191-197.

Williams, J.E. and Whittemore, A.D. (1971) Serological diagnosis of pullorum disease with the micro-agglutination system. Appl. Microbiol., 21: 392-399.

\section{How to cite this article:}

Anju Mohan and Hari Mohan Saxena. 2018. Analysis of Sera from Cattle Suspected for Brucellosis by Agglutination Tests and ELISA. Int.J.Curr.Microbiol.App.Sci. 7(11): 351-362. doi: https://doi.org/10.20546/ijcmas.2018.711.044 